\title{
A perspective on atomically thin $2 d$ inorganic layered materials for biosensor
}

\begin{abstract}
The 2D inorganic materials have ability to play important role in the designing of novel biosensor devices for the future. The advantages are originated from their unique electronic and optical properties along with large area available for the biofunctonalization. We have attempted to address few challenges and issues for developing biosensors based on 2D materials for practical applications for enhanced life time, improved durability, and biocompatibility. Use of 2D nanocomposites materials and doping them using various noble metals will be very exciting and challenging areas of research for future biosensor to be explored to achieve selectivity and high sensitivity with very low detection limit. In next phase of structural DNA/RNA nanotechnology in combination with $2 \mathrm{D}$ inorganic layered materials, novel interactions can expected between DNA, RNA, 2D inorganic layered materials and proteins. This has direct relevance to develop new molecular design, computing facility, programming and assembly of the new functional materials.
\end{abstract}

Volume 2 Issue I - 2015

\author{
Dattatray J Late,' Chandra Sekhar Rout ${ }^{2}$ \\ 'Physical \& Materials Chemistry Division, CSIR National \\ Chemical Laboratory, India \\ ${ }^{2}$ School of Basic Sciences, Indian Institute of Technology, India
}

Correspondence: Dattatray J Late, Physical \& Materials

Chemistry Division, CSIR National Chemical Laboratory, India Email datta099@gmail, dj.late@ncl.res.in;csrout@litbbs.ac.in

Received: December 09, 2014 | Published: December 30 2014

Keywords: Layered materials, Biosensor, DNA, RNA

\section{Introduction}

Graphene has attracted much attention in recent years due to many potential proof-of-concept device demonstrations; such as transistors. solar cells. ${ }^{2}$ and especially gas sensors. ${ }^{3}$ and biosensors. ${ }^{4}$ For gas sensors, the ancient experience of conventional semiconducting metal oxides indicates improved performance with increase in the surfaceto-volume ratio, unique physical and chemical properties, excellent electrical conductivity, good chemical stability and strong mechanical strength. This has inspired scientific community to use graphene a one-atomically thin 2D materials which has exceedingly high surfaceto-volume ratio and is believed to outperform traditional sensors and biosensor. Indeed, there are reports on graphene which shows good sensing. ${ }^{3}$ and biosensing performance. ${ }^{4}$ However, other than surfaceto-volume ratio, other significant factors for a good sensor include the semiconducting nature of the materials and easy accessibility for the reactive sites for the redox reactions. Graphene due to its limitation as band gap material hinders its wide applications. Therefore graphene analogues of transition metal dichalcogenides (TMDCs) inorganic layered materials have been widely emerged as alternative materials due the appropriate band gap. Also, many interesting and exceptional properties appropriate for sensing applications can be realized by integrating them into composite and hybrids assemblies. To date several inorganic 2D TMDCs layered materials such including $\mathrm{MoS}_{2}{ }^{5-}$ ${ }^{8} \mathrm{WS}_{2}{ }^{9-10} \mathrm{MoSe}^{11,12} \mathrm{WSe}_{2}{ }^{11}$ and III-VI group semiconducting layered materials $\mathrm{GaS}$ and GaSe. ${ }^{13}$ etc. reported and identified for possible nanoelectronic device applications. Further, most of literature reports do not emphasize on the subtleties of gas and biosensing performance and its relation to layered structures but instead demonstrate only gas sensing or biosensing as a proof-of-concept. ${ }^{3,8,12}$ There are very few attempts on few of these materials for bio sensor applications. ${ }^{14,15}$

The biosensors field has been under progress for the last 45 years and the research in this field has become very popular since last 25 years. Biosensor is nothing but a device where a biological recognition element is built in (physically attached or confined) and is the primary selectivity element. Biosensors are also known as biochips, immune sensors, glucometers, biocomputers, optrodes, chemical canaries, resonant mirrors and so on. Many sensors used for biological purposes are therefore not biosensors, including those for temperature, pressure, electrocardiograms, $\mathrm{pH}, \mathrm{Ca}^{2+}$, catecholamine's and the like. ${ }^{16}$ Compared to other traditional analytical techniques, biosensors show several advantages such as high specificity, high sensitivity and low cost. In biosensor devices, recognition elements play a vital role in the detection of electro active species. Examples of some of the bio-recognition elements are enzymes, micro-organisms, nucleic acids, cells and anti-bodies. Among these, enzymes are widely used in electrochemical biosensors. Much of the biosensors so far reported employed electrochemical techniques in which the electrical properties of the bio-system are extracted by using a suitable biorecognition element. Various electrochemical techniques such as amperometry, potentiometry and cyclic voltametry have been widely used in the development of electrochemical biosensors. Recently, impedance based electrochemical biosensors are emerging because of its ability to detect very low concentration of species at ppb level. Other than electrochemical approaches, some of the techniques like optical, gravimetric are being employed in biosensing application. During the past few years, extensive efforts have been taken for developing biosensors with high sensitivity and low detection limit using various nanostructured materials.

The use of layered inorganic nanomaterials in the development of biosensors is recently emerging because of its remarkable electrical and optical properties which are highly dependent on the number of layers present on the materials. ${ }^{5,6}$ The surface to volume ratio of the layered materials is also dependent on the number of layers. Therefore, one can tune the sensitivity parameters by changing the number of layers present in the materials. Among the various transduction techniques, electrochemical methods are so far widely studied and employed for the development of biosensors. Various 2D layered inorganic materials including $\mathrm{MoS}_{2}, \mathrm{WS}_{2}, \mathrm{SnS}_{2}$ and $\mathrm{CuS}$ have been used in electrochemical biosensing for the detection of analytes such as glucose, dopamine, hydrogen peroxide and DNA. ${ }^{14,15}$ Enzymes like glucose oxidase, horseradish peroxide have been used as bio recognition elements. Apart from simple layered materials, recently their nanocomposites with gold, chitosan and carbon nanotube have been used in biosensing. ${ }^{17}$

Even though, there have been some reports are available on the use of 2D layered inorganic materials and its nanocomposite in 
biosensing, still there are some challenges remains to be solved. ${ }^{17}$ In most of the reported literature, active materials are chemically synthesized and drop casted on the surface of electrode for sensing application. Except one or two reports on one-time preparation of layered materials on the electrode surface is a challenging, as it avoids the large consumption of time. The use of hybrid layered materials on the development of biosensors is another interesting area because of its synergetic relationship. Other than metal nanoparticles, some of the materials like conducting polymers, metal oxides and mixed metal oxides along with layered inorganic materials need to be prepared and employed in biosensing. The modification of electrode surface by noble metal nanoparticles will actually fasten the electron transfer ability in the electrode/electrolyte interface. Thus, the application of various noble metal particles on the modification of electrode surface will actually pave the way for the development of electrochemical biosensors with high sensitivity and selectivity. Figure 1 shows the typical schematic of a reduced graphene oxide transistor to detect specific protein anti-IgG through Au nanoparticle and functions as a specific recognition group for the IgG binding. The electrical signal detection of the protein binding ( $\operatorname{IgG}$ to anti-IgG) is consummate by transistor characteristics. ${ }^{18}$

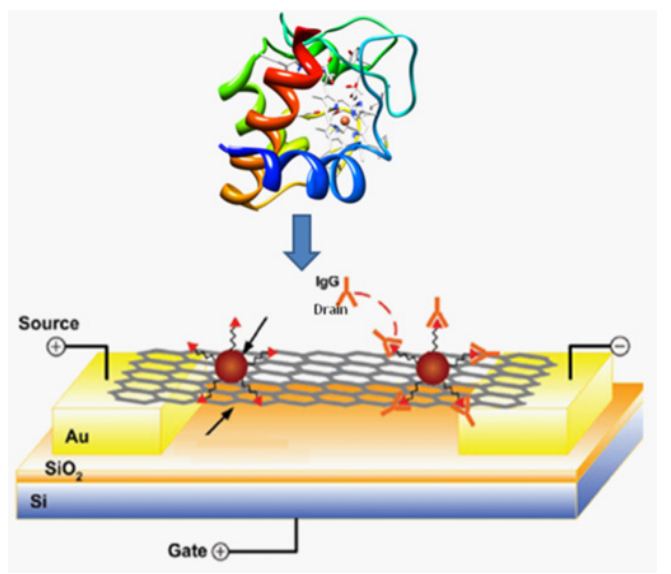

Figure I Schematic of a reduced graphene oxide transistor. The specific protein anti-lgG is anchored to the graphene sheet surface through Au nanoparticle and functions as a specific recognition group for the IgG binding. The electrical signal detection of the protein binding (IgG to anti$\operatorname{lgG}$ ) is consummate by transistor characteristics. Top figure is Cytochrome c which is highly soluble protein. [Reprinted with permission from ref.$^{18}$ Copyright 2010 Wiley-VCH].

The information-rich character of self-assembling DNA / RNA nanostructures in combination with 2D layered materials can generate several new frontiers for the application of designer DNA/RNA nanostructures for various applications including sensors, computers, molecular scaffolds and robots..$^{20}$ As 30 years history of this research field growth, structural DNA/RNA nanotechnology is shifting from infancy into maturity. The research field now crossing the borders of materials science, Physics, Chemistry, Biology, Nanotechnology and engineering and is balanced to produce exceptional ways and solutions to the real world problems in science and new generation technology. ${ }^{20}$ It is expected that in next phase of structural DNA / RNA nanotechnology in combination with these 2D inorganic layered materials, novel interactions can takes place between DNA, RNA, 2D materials and proteins, this can be used to make probable the nanotechnology, which can sustain the major opportunities and challenges for developing new molecular design, computing, programming and assembly. ${ }^{18-21}$

\section{Conclusion and future outlook}

This article summarized the recent progresses of $2 \mathrm{D}$ inorganic layered nano sheets materials for biosensing applications. Among the advantages of 2D inorganic layered materials over the carbon based materials is due to its finite band gap and tuning of its physical and chemical properties depending on our requirements. The 2D inorganic materials will play important role in designing novel biosensor devices for the future due to their unique properties and large area available for biofunctonalization. A major challenge in developing biosensors for practical applications needs to have enhanced life time, improved durability, and biocompatibility. Also determination of any possible toxicity and health risks during long term use needs to be explored. Application of nanocomposites of 2D materials and effect of noble metals such as $\mathrm{Au}, \mathrm{Pt}$ and Pd etc and doping effects need to be explored to achieve selectivity and high sensitivity with very low detection limit. It is expected that in next phase of structural DNA/ RNA nanotechnology in combination with these 2D inorganic layered materials, novel interactions can takes place between DNA, RNA, 2D materials and proteins, this can be used to for developing new molecular design, computing, programming and assembly. However, the investigation of 2D inorganic layered materials for biosensing is still at their premature stage, and several unsolved significant issues are to be addressed to further make possible the advances of this emerging and challenging area of research. These layered materials also have great demand and potentials in other bio related applications including the gene therapy, tissue engineering and radio sensitization targeting strategy for high therapeutic/diagnostic performance.

\section{Acknowledgement}

The work was supported by Department of Science and Technology (Government of India) for Ramanujan Fellowship to Dr. D. J. Late (Grant No. SR/S2/RJN-130/2012), NCL-MLP project grant 028626, DST-SERB Fast-track Young scientist project Grant No. SB/FT/CS$116 / 2013$ and the partial support by INUP IITB project sponsored by DeitY, MCIT, Government of India. Dr. C.S. Rout thanks DST-SERB and UGC-UKIERI for the research grants (Grant No. SB/FTP/PS065/2013, SR/S2/RJN-21/2012 and UGC-2013-14/005).

\section{Conflict of interest}

None.

\section{References}

1. Novoselov KS, Geim AK, Morozov SV et al. Electric Field Effect in Atomically Thin Carbon Films. Science. 2004;306(5696):666-669.

2. Wang JT, Ball JM, Barea EM et al. Low-Temperature Processed Electron Collection Layers of Graphene/ $\mathrm{TiO}_{2}$ Nanocomposites in Thin Film Perovskite Solar Cells. Nano Lett. 2014;14(2):724-730.

3. Ghosh A, Late DJ, Panchakarla LS et al. $\mathrm{NO}_{2}$ and Humidity Sensing Characteristics of Few-Layer Graphene. J Exp Nanosci. 2009;4:313-322.

4. Wang $\mathrm{T}$, Zhu H, Zhuo J et al. Biosensor Based on Ultra small $\mathrm{MoS}_{2}$ Nanoparticles for Electrochemical Detection of $\mathrm{H}_{2} \mathrm{O}_{2}$ Released by Cells at the Nano molar Level. Anal Chem. 2013;85(21):10289-10295.

5. Matte HS, Gomathi A, Manna AK et al. $\mathrm{MoS}_{2}$ and $\mathrm{WS}_{2}$ Analogues of Graphene. Angew Chem Int Ed Engl. 2010;49(24):4059-4062.

6. Late DJ, Liu B, Matte HSSR et al. Rapid Characterization of Ultrathin Layers of Chalocogenides on $\mathrm{SiO}_{2} / \mathrm{Si}$ Substrates. Adv Fun Mater. 2012;22(9):1894-1905.

7. Late DJ, Liu B, Matte HS et al. Hysteresis in Single-Layer $\mathrm{MoS}_{2}$ Field Effect Transistors. ACS Nano. 2012;6(6):5635-5641. 
8. (a)Late DJ, Huang YK, Liu B et al. Sensing behavior of atomically thinlayered MoS2 transistors. ACS Nano. 2013;7(6):4879-4891.

9. (b)Late DJ, Shaikh PA, Khare R et al. Pulsed laser-deposited $\mathrm{MoS}_{2}$ thin films on W and Si: field emission and photo response studies. ACS Appl Mater Interfaces. 2014;6(18):15881-15888.

10. Thripuranthaka M, Kashid RV, Rout CS et al. Temperature dependent Raman spectroscopy of chemically derived few layer MoS2 and WS2 nano sheets. Appl Phy Lett. 2014;104(8):081911.

11. M T, Late DJ Temperature dependent phonon shifts in single-layer WS(2). ACS Appl Mat Interfaces. 2014;6(2):1158-1163.

12. Late DJ, Shirodkar SN, Waghmare UV et al. Chemphyschem. 2014;15(8):1592-1598.

13. Late DJ, Doneux T, Bougouma M Single-layer MoSe2 based NH3 gas sensor. Appl Phy Lett. 2014;105(23):233103.

14. Late DJ, Liu B, Luo J et al. GaS and GaSe Ultrathin Layer Transistors. Adv Mater. 2012;24(26): 3549-3554.

15. Xi Q, Zhou DM, Kan YY et al. Highly Sensitive and Selective Strategy for Micro RNA Detection Based on WS $\mathrm{W}_{2}$ Nano sheet Mediated Fluorescence
Quenching and Duplex-Specific Nuclease Signal Amplification. Anal Chem. 2014;86(3):1361-1365.

16. Yuan Y, Li R, Liu Z Establishing Water-Soluble Layered WS Nano sheet as a Platform for Biosensing. Anal Chem. 2014;86(7):3610-3615.

17. Kissinger PT Biosensors a perspective. Biosens Bioelectron. 2005;20(12):2512-2516.

18. Huang KJ, Liu YJ, Wang HB et al. Sub-femto molar DNA detection based on layered molybdenum disulfide/multi-walled carbon nanotube composites, Au nanoparticle and enzyme multiple signal amplification. Biosens Bioelectron. 2014;55:195-202.

19. Mao S, Lu G, Yu K et al. Specific Protein Detection Using Thermally Reduced Graphene Oxide Sheet Decorated with Gold NanoparticleAntibody Conjugates. Adv Mater. 2010;22(32):3521-3526.

20. Zhang F, Nangreave J, Liu Y et al. Structural DNA Nanotechnology: State of the Art and Future Perspective. $J$ Am Chem Soc. 2014;136(32):11198-11211.

21. Chen Y, Tan C, Zhang H et al. Two-dimensional graphene analogues for biomedical applications. Chem Soc Rev. 2014. 